\title{
Prediction of malignant sinonasal inverted papilloma transformation by preoperative computed tomography and magnetic resonance imaging*
}

\author{
Lisong Zhang 1,2,3,\#, Gaoli Fang 3,\#, Wenling Yư ${ }^{4}$, Bentao Yang ${ }^{4}$, \\ Chengshuo Wang', Luo Zhang ${ }^{1,2}$ \\ ' Department of Otolaryngology Head and Neck Surgery, Beijing TongRen Hospital, Capital Medical University, Beijing, PR China \\ ${ }^{2}$ Beijing Key Laboratory of nasal diseases, Beijing Institute of Otolaryngology, Beijing, PR China \\ ${ }^{3}$ Department of Otolaryngology Head and Neck Surgery, Beijing DiTan Hospital, Capital Medical University, Beijing, PR China \\ ${ }^{4}$ Department of Radiology, Beijing TongRen Hospital, Capital Medical University, Beijing, PR China
}

Rhinology 58: 3, 248 - 256, 2020

https://doi.org/10.4193/Rhin19.240

*Received for publication:

July 11, 2019

Accepted: December 15, 2019

\# These authors contributed equally to the study

\begin{abstract}
Background: Accurate preoperative prediction of the malignant transformation of sinonasal inverted papilloma (SNIP) is essential for radical resection of tumours and prevention of recurrence. We here explored the predictive value of preoperative computed tomography (CT) and magnetic resonance imaging (MRI).
\end{abstract}

Methodology: The study was performed on 268 patients with SNIP with $(n=78)$ or without $(n=190)$ coexistent malignant transformation. We used univariate and multivariate logistic regression analysis method to screen for independent risk factors, and established a nomogram model. Finally, using receiver operating characteristic curves, we assessed the diagnostic value of the independent risk factors for malignant transformation of SNIP.

Results: We identified bone erosion on CT, change in convoluted cerebriform pattern (CCP) on MRI, and washout-type timeintensity curve (TIC) of dynamic contrast-enhanced (DCE)-MRI were independent predictors of malignant transformation of SNIP. The scores of these three independent risk factors from the nomogram model were 10, 7 and 8, respectively. The area under the receiver operating characteristic curve for predicting SNIP malignancy was 0.954 for the nomogram model, 0.826 for bone erosion, 0.776 for washout-type TIC, and 0.810 for CCP mutation.

Conclusions: Of the independent risk factors and related combination identified, the nomogram model based on bone destruction on CT, CCP mutation on MRI, and washout-type TIC of DCE-MRI had the best predictive value for malignant transformation of SNIP.

Key words: bone erosion, computed tomography, magnetic resonance imaging, malignant transformation, predictive factor, sinonasal inverted papilloma

\section{Introduction}

Sinonasal inverted papilloma (SNIP) is a common benign sinonasal epithelial neoplasm, with a high recurrence rate and the possibility of association with squamous cell carcinoma (SCC) ${ }^{(1,2)}$; the incidence of association with SCC is approximately $6.5-10 \%^{(3-6)}$. The clinical signs and symptoms caused by SNIP- transformed squamous cell carcinomas (SNIP-SCC) are of little diagnostic value. Local biopsy has typically been recommended in previous studies, because most malignancies expand from the center of the SNIP (7). However, these have low diagnostic accuracy due to the uncertainty of whether the correct tissue was sampled, if the tumour is large or located in a position that is not 
easily visible by nasal endoscopy ${ }^{(8)}$. These difficulties hinder the administration of appropriate therapeutic strategies. Therefore, presurgical distinction between SNIP-SCC and SNIP, although difficult, is important.

Previous studies of the difference between computed tomography (CT) and magnetic resonance imaging (MRI) manifestations of SNIP and malignant tumours have found that bony erosion on $\mathrm{CT}$, involvement of adjacent structures, and intermediate signal intensity on T2-weighted (T2W) MRI sequences are the hallmarks of sinonasal malignant tumours ${ }^{(9-15)}$. Convoluted cerebriform pattern (CCP) is a classical and distinctive MRI feature of inverted papilloma ${ }^{(13-17)}$. One previous study on MRI considered that focal disappearance of a CCP might forebode the presence of malignant transformation ${ }^{(15)}$. CCP change includes the disappearance and disorder of CCP and can be divided into partial mutation and total mutation. It would be helpful to predict the possibility of SNIP-SCC upon total mutation in cases with a history of diagnosis of SNIP and CCP on MR images. Dynamic contrast-enhanced MRI (DCE-MRI) is an imaging tool that helps to differentiate SNIP-SCC from SNIP. Jeon et al. ${ }^{(18)}$ reported that CCP was observed in all 30 (100\%) of the inverted papilloma (IPs) and in 17 (13\%) of the 128 malignant sinonasal tumours studied; in their study, the washout-type time-intensity curve (TIC) of DCE-MRI was the imaging feature that could best distinguish SNIP-SCCs from SNIP ${ }^{(19)}$.

A nomogram is a two-dimensional diagram that allows graphical calculations, which could offer a more individualized and precise prediction compared to traditional models for various diseases ${ }^{(20-23)}$, but has not been utilized to predict malignancy of SNIP to date. This study sought to determine the risk factors of malignant transformation of SNIP from CT and MR images, to produce a prognostic nomogram, and to evaluate whether this could be of benefit for predicting malignancy of SNIP in a clinical setting.

\section{Material and methods}

\section{Study design}

In total, 268 patients hospitalized at Beijing Tongren Hospital affiliated with Capital Medical University, between June 2015 and July 2018, with SNIP with $(n=78)$ or without $(n=190)$ coexistent malignant transformation, confirmed by pathological findings, were enrolled in the study. CT or MRI with or without DCE-MRI scans was performed preoperatively in each patient. Some patients were imaged two or three times; recurrent tumours were included in the study.

Study approval was granted by the Institutional Review Board of Beijing TongRen Hospital and informed consent was obtained from all patients for review of images.

Preoperative examination

CT imaging protocol
Preoperative unenhanced paranasal sinus CT examinations were performed with a Lightspeed 16-section CT system (GE Healthcare, Chicago, IL, USA) or a Brilliance 64-section CT system (Phillips, Best, the Netherlands). Images were obtained in the axial, coronal, and sagittal planes. All CT images, reconstructed by using a bone algorithm, were observed in a bone window setting. The imaging parameters were as follows: voltage, $120 \mathrm{kV}$; current, $200 \mathrm{~mA}$; matrix, $512 \times 512$; and section thickness, $2 \mathrm{~mm}$.

\section{MRI protocol}

MRI examinations were performed using an 8-channel phasedarray head coil on a GE Excite 1.5-T MR or a GE HDxt 3-T MR scanner (GE Healthcare). Precontrast axial and coronal T1-weighted (T1W) spin-echo images and T2W fast spin-echo images were obtained. Then, we injected $0.1 \mathrm{mmol} / \mathrm{kg}$ of gadopentetate dimeglumine into the vein, after which contrast-enhanced axial, coronal, and sagittal T1W spin-echo images were obtained. Frequency-selective fat saturation in contrast-enhanced T1W imaging was performed in the axial and coronal planes. Imaging parameters were set at a section thickness of 4-5 mm, with an intersection gap of $0.5 \mathrm{~mm}$, a matrix of $320 \times 256$, and field of view $20 \times 20 \mathrm{~cm}$.

DCE-MRI used a fast spoiled gradient-echo order and a matrix of $256 \times 160$. Twelve sets of dynamic images were acquired with the 1.5-T scanner. Each scan, which included 12 sections, lasted 13 seconds, and the interval between two scans was 12 seconds. Total scanning time was about 288 seconds. Thirty-seven scans were acquired with the 3-T scanner. For each scan, 16 9-second sections were acquired, with an interval of $0-2$ seconds between two scans. The total scanning time was 344 seconds. Gadopentetate dimeglumine was administered intravenously at a flow velocity of $2 \mathrm{ml} / \mathrm{s}$ with a power injector.

\section{Image postprocessing and interpretation}

CT features of the important soft tissue, bone erosion, and the relationship with surrounding structures, particularly the extrasinonasal structures, were investigated. The site of bone erosion was defined as the extra-sinonasal structures, especially the orbital and intracranial invasion. MRI features of interest were related to the intensity of $\mathrm{T} 2$ signals compared to grey matter, CCP on T2W or contrast-enhanced T1W imaging, and extra-sinonasal involvement. Multiphase dynamic images were analysed using a GEAW 4.4 workstation. A region-of-interest (ROI) of 10 $\mathrm{mm}^{2}$ that manifested the greatest degree of early enhancement was assessed. When the time-to-peak enhancement (Tpeak) was longer than 120 seconds, we termed it persistent-type TIC. When the Tpeak was shorter than or equal to 120 seconds and the washout ratio (WR) was less than or equal to $15 \%$, we termed it plateau-type TIC. When the Tpeak was shorter than or equal to 120 seconds and WR was greater than $15 \%$, we termed it washout-type TIC. 
Table 1. Imaging characteristics of the enrolled SNIP and SNIP-SCC.

\begin{tabular}{|c|c|c|c|c|c|}
\hline Imaging features & Total N = 268 & SNIP N = 190 & SNIP-SCC N = 78 & $x^{2}$ & p value \\
\hline $\begin{array}{l}\text { Extranasal invasion (CT, MRI) } \\
\text { No } \\
\text { Yes }\end{array}$ & $\begin{array}{l}188(70.15) \\
80(29.85)\end{array}$ & $\begin{array}{c}169(88.95) \\
21(11.05)\end{array}$ & $\begin{array}{l}19(24.36) \\
59(75.64)\end{array}$ & 110.17 & $<0.001$ \\
\hline $\begin{array}{l}\text { Bone erosion }(\mathrm{CT}) \\
\text { No } \\
\text { Yes }\end{array}$ & $\begin{array}{l}155(67.98) \\
73(32.02)\end{array}$ & $\begin{array}{l}142(87.65) \\
20(12.35)\end{array}$ & $\begin{array}{l}13(19.70) \\
53(80.30)\end{array}$ & 99.50 & $<0.001$ \\
\hline $\begin{array}{l}\text { T2 equal signal (MRI) } \\
\text { No } \\
\text { Yes }\end{array}$ & $\begin{array}{c}180(72.29) \\
69(27.71)\end{array}$ & $\begin{array}{c}150(81.08) \\
35(18.92)\end{array}$ & $\begin{array}{l}30(46.88) \\
34(53.13)\end{array}$ & 27.77 & $<0.001$ \\
\hline $\begin{array}{l}\text { CCP mutation (MRI) } \\
\text { No } \\
\text { Yes }\end{array}$ & $\begin{array}{l}175(70.28) \\
74(29.72)\end{array}$ & $\begin{array}{c}158(85.41) \\
27(14.59)\end{array}$ & $\begin{array}{l}17(26.56) \\
47(73.44)\end{array}$ & 78.83 & $<0.001$ \\
\hline $\begin{array}{l}\text { Washout-type TIC (DCE-MRI) } \\
\text { No } \\
\text { Yes }\end{array}$ & $\begin{array}{c}140(76.92) \\
42(23.08)\end{array}$ & $\begin{array}{c}119(93.70) \\
8(6.30)\end{array}$ & $\begin{array}{l}21(38.18) \\
34(61.82)\end{array}$ & 66.64 & $<0.001$ \\
\hline
\end{tabular}

There were significant differences in each index between the two group.

SNIP = Sinonasal inverted papilloma; SNIP-SCC = SNIP-transformed squamous cell carcinomas; $C$ T = Computed tomography; $\mathrm{MRI}=$ Magnetic resonance imaging; DCE-MRI = Dynamic contrast-enhanced MRI; CCP = Convoluted cerebriform pattern; TIC = time-intensity curve .

All CT and MR images, including DCE-MRI, were evaluated independently by two radiologists who had more than 8 years of experience in head and neck imaging. They were blinded to the patient's medical history and diagnosis. If they disagreed on the assessment of a section of the image, they would re-evaluate the section to obtain a consistent conclusion. All the images were observed on OsiriX version 5.8.1 (Pixmeo, Switzerland) in DICOM format.

\section{Statistical analysis}

Statistical analysis was conducted using SPSS version 19.0 (IBM SPSS, Armonk, NY, USA). For continuous quantitative variables, mean \pm standard deviation was used to describe the difference between groups, and the t-test was used to assess the difference between groups. For qualitative variables, frequency and percentage were used as descriptive statistics, and component differences were tested by $\chi^{2}$ test or Fisher's exact test. A nomogram model was built and the score for predicting malignancy of SNIP was calculated according to results of multivariate logistic regression analyses using $R$ version 3.0.2 with the rms package ${ }^{(24)}$. A backward step-down selection process was applied to the selection of risk factors of the final model. The ability of the nomogram to predict malignant transformation of SNIP was evaluated using receiver operating characteristic (ROC) curves. Finally, by comparing the area under the ROC curve (AUC), $95 \%$ confidence interval, sensitivity, and specificity of various indicators, related combinations, and the nomogram model, the factors were assessed for their ability to predict malignant transformation of SNIP. P $<0.05$ was considered to be statistically significant.

\section{Results}

\section{Patient characteristics and outcomes}

Age and whether the nasal cavity or maxillary sinus was involved differed between SNIP-SCC and SNIP. The average age of SNIP patients was $50.63 \pm 13.06$ years, and $54.32 \pm 14.24$ years in SNIP-SCC group ( $p<0.05)$. The incidence of nasal cavity involvement was $84.74 \%$ in SNIP and $73.08 \%$ in SNIP-SCC ( $p<$ 0.05 ), while maxillary sinus involvement was $39.47 \%$ in SNIP and $52.56 \%$ in SNIP-SCC $(p<0.05)$. The occurrence of malignant transformation was $21.21 \%(28 / 132)$ in the initial group and $36.76 \%(50 / 136)$ in the recurrent group $(p<0.01)$. There were also significant differences in extra-sinonasal invasion, bone erosion, $\mathrm{T} 2$ equal signal, CCP mutation, and the washout-type TIC between the two groups $(p<0.01)$ (Table 1). There was no difference in gender; side; single or multiple lesions; whether the ethmoid sinus, frontal sinus, and sphenoid sinus were involved; or whether there was sinus involvement between the two groups ( $p>0.05)$.

\section{Univariate and multivariate logistic analysis} Univariate logistic analysis showed that older age, recurrence, lack of involvement of the nasal cavity, extranasal invasion, bone erosion, $\mathrm{T} 2$ equal signal, CCP mutation, and washout-type TIC were significantly associated with malignant transformation of SNIP $(p<0.05$, Table 2$)$.

A backward step-down selection process was used to screen the variables by multivariate logistic regression analysis. The results suggested that only bone erosion (Figure 1) $(\mathrm{OR}=38.814,95 \%$ Cl: 9.273-162.454, p < 0.001), CCP mutation (Figure 2) (OR= 12.089, 95\% Cl:3.381-43.226, p < 0.001), and washout-type TIC 
Table 2. Univariate logistic regression analysis of malignant transformation of SNIP.

\begin{tabular}{|c|c|c|c|c|c|c|c|}
\hline & \multirow{2}{*}{ b } & \multirow{2}{*}{ stb } & \multirow{2}{*}{ Wald $\chi^{2}$} & \multirow{2}{*}{$\mathbf{P}$} & \multirow{2}{*}{ OR } & \multicolumn{2}{|c|}{$95 \% \mathrm{Cl}$ for OR } \\
\hline & & & & & & Lower & Upper \\
\hline Male sex & -0.049 & 0.294 & 0.028 & 0.867 & 0.952 & 0.535 & 1.694 \\
\hline Age (years) & 0.021 & 0.010 & 4.085 & 0.043 & 1.021 & 1.001 & 1.042 \\
\hline Recurrence & 0.770 & 0.277 & 7.701 & 0.006 & 2.159 & 1.254 & 3.719 \\
\hline \multicolumn{8}{|l|}{ Side } \\
\hline Right/Left & 0.049 & 0.277 & 0.032 & 0.858 & 1.051 & 0.610 & 1.808 \\
\hline Bilateral/Unilateral & 0.608 & 0.618 & 0.967 & 0.325 & 1.837 & 0.547 & 6.174 \\
\hline \multicolumn{8}{|l|}{ Location } \\
\hline Multiple sites/Single site & -0.156 & 0.271 & 0.330 & 0.565 & 0.856 & 0.503 & 1.456 \\
\hline Nasal cavity & -0.716 & 0.325 & 4.840 & 0.028 & 0.489 & 0.258 & 0.925 \\
\hline Maxillary sinus & 0.530 & 0.271 & 3.826 & 0.050 & 1.699 & 0.999 & 2.890 \\
\hline Ethmoid sinus & 0.071 & 0.289 & 0.060 & 0.806 & 1.074 & 0.609 & 1.893 \\
\hline Frontal sinus & 0.282 & 0.334 & 0.711 & 0.399 & 1.326 & 0.688 & 2.554 \\
\hline Sphenoid sinus & -0.328 & 0.672 & 0.238 & 0.625 & 0.720 & 0.193 & 2.690 \\
\hline Extranasal invasion & 3.218 & 0.351 & 84.136 & 0.000 & 24.990 & 12.563 & 49.709 \\
\hline Bone erosion & 3.365 & 0.391 & 74.108 & 0.000 & 28.946 & 13.453 & 62.281 \\
\hline T2 equal signal & 1.580 & 0.313 & 25.492 & 0.000 & 4.857 & 2.630 & 8.971 \\
\hline CCP mutation & 2.784 & 0.351 & 62.762 & 0.000 & 16.179 & 8.125 & 32.213 \\
\hline Washout-type TIC & 3.181 & 0.459 & 48.100 & 0.000 & 24.082 & 9.800 & 59.179 \\
\hline
\end{tabular}

According to the results of univariate Logistic regression analysis, extranasal invasion, bone erosion, T2 equal signal, CCP mutation and Washout-type $\mathrm{TIC}$ had significant differences between the two groups. $\mathrm{OR}=$ odds ratio; $\mathrm{Cl}=$ confidence interval; $\mathrm{CCP}=$ Convoluted cerebriform pattern; $\mathrm{TIC}=$ timeintensity curve.

Table 3. Multivariate Logistic regression analysis of malignant transformation of SNIP.

\begin{tabular}{|c|c|c|c|c|c|c|c|}
\hline & \multirow{2}{*}{ b } & \multirow{2}{*}{ stb } & \multirow{2}{*}{ Wald $x^{2}$} & \multirow{2}{*}{$\mathbf{P}$} & \multirow{2}{*}{ OR } & \multicolumn{2}{|c|}{$95 \% \mathrm{Cl}$ for OR } \\
\hline & & & & & & Lower & Upper \\
\hline Intercept & -4.678 & 0.777 & 36.274 & 0.000 & & & \\
\hline Bone erosion & 3.659 & 0.730 & 25.091 & 0.000 & 38.814 & 9.273 & 162.454 \\
\hline CCP mutation & 2.492 & 0.650 & 14.697 & 0.000 & 12.089 & 3.381 & 43.226 \\
\hline Washout-type TIC & 2.827 & 0.739 & 14.630 & 0.000 & 16.899 & 3.969 & 71.951 \\
\hline
\end{tabular}

According to the results of multivariate Logistic regression analysis, only the three factors above were finally identified as risk factors for malignant transformation of SNIP. OR = odds ratio; $\mathrm{Cl}=$ confidence interval; $\mathrm{CCP}=$ convoluted cerebriform pattern; $\mathrm{TIC}=$ time-intensity curve.

(Figure 3) $(\mathrm{OR}=16.899,95 \% \mathrm{Cl}: 3.969-71.951, \mathrm{p}<0.001)($ Table 3) were independent risk factors for predicting malignancy of SNIP.

\section{Construction of prognostic nomogram}

A nomogram was established based on the coefficients gained from multivariate logistic regression analysis, which included bone erosion, washout-type TIC, and CCP mutation (Figure 4). Assuming that the score for bone erosion, the strongest risk factor, was 10, the scores of other risk factors could be calculated: the score for the washout-type TIC was 8 and that of CCP mu- tation was 7. According to the scoring chart nomogram model, each of the indicators was allocated a score on the point-scale axis. According to the score of 5, 7, 13, 16, and 21 points predicted by the model, the corresponding risk of SNIP canceration is $5 \%, 10 \%, 50 \%, 75 \%$, and $95 \%$ respectively. By summing each single score and using that value in the total point-scale axis, the total score could be easily calculated to assign the probability of malignant transformation of SNIP for individual patients.

Comparison of ROC curves for predicting malignant transfor- 


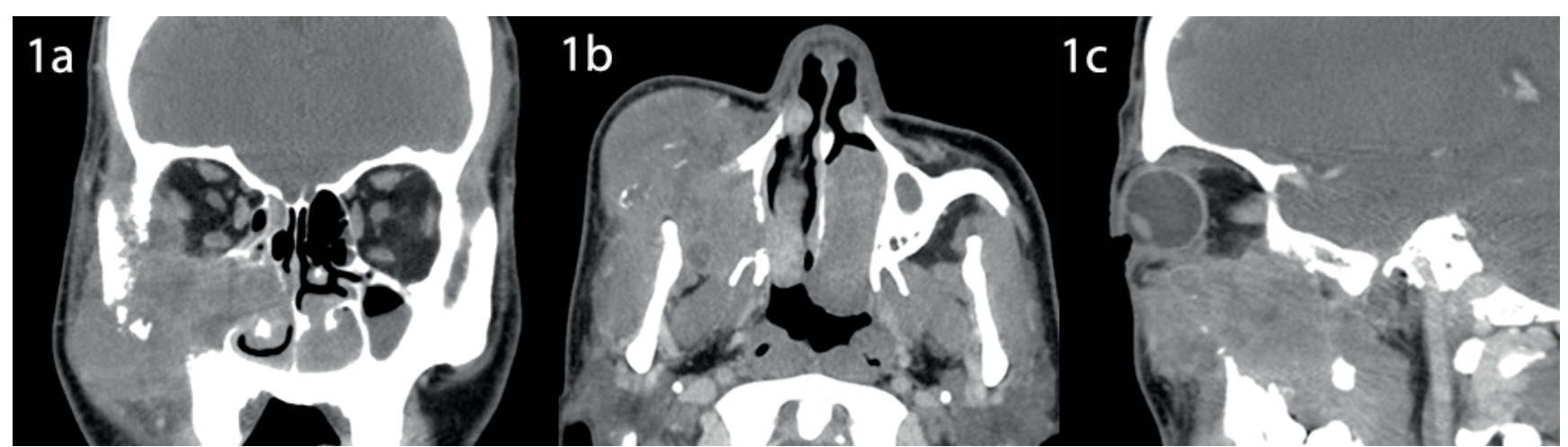

Figure 1. Coronal (a), axial (b), and sagittal (c) positions CT examination was performed on a 48-year-old male patient with primary SNIP-SCC. Irregular soft tissue focus, which mainly located in the right maxillary sinus invaded adjacent bone on CT. Lesions invade the sinus walls with erosion of adjacent bone and damage the corresponding tissues and organs (superior wall into the orbit, medial wall into the nasal cavity, posterior/lateral wall into the pterygopalatine fossa and lateral pterygoid muscle, inferior wall into the arcus alveolaris, anterior /lateral wall into the nasal-facial soft tissue, ductus nasolacrimalis and zygomatic process). CT = computed tomography; SNIP = sinonasal inverted papilloma; SNIP-SCC = SNIP-transformed squamous cell carcinomas.

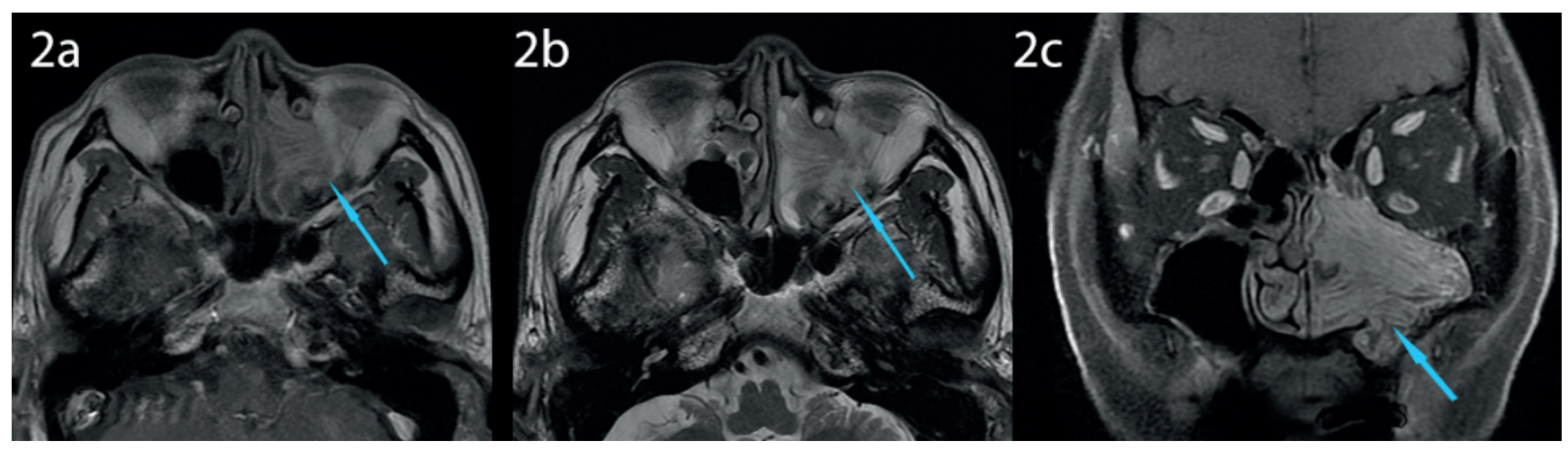

Figure 2. Axial (2a, contrast-enhanced T1WI), Axial (2b, T2WI), and coronal (2c, contrast-enhanced T1WI) MRI examination was performed on a 67-yearold male patient with SNIP-SCC having undergone surgery. The lesions located in the left middle inferior nasal meatus and maxillary ostium with regular CCP. CCP mutation (blue arrows) was found near the left posterolateral wall of the maxillary sinus, which was confirmed to be SNIP-SCC by pathologic examination. $\mathrm{CCP}=$ convoluted cerebriform pattern; SNIP = sinonasal inverted papilloma; SNIP-SCC = SNIP-transformed squamous cell carcinomas.

mation of SNIP with a nomogram consisting of three risk factors individually and in combination

The ROC was drawn with the scoring data of the nomogram, bone erosion, CCP mutation, washout-type TIC, and the combination of these factors. The AUCs were used to judge the predictive value of the nomogram model, these indicators, and the relevant combinations. The AUC of the nomogram was 0.954, which was significantly higher than those of bone erosion (0.826), CCP mutation (0.810), washout-type TIC (0.776), bone erosion + CCP mutation (0.808), bone erosion+ washout-type TIC (0.730), washout-type TIC + CCP mutation (0.738), and bone erosion + washout-type TIC + CCP mutation $(0.694)(p<0.001$ for all). This indicates that the nomogram had superior clinical usefulness for predicting SNIP-SCC over other models (Figures $5,6)$

\section{Discussion}

It is crucial to predict malignant tumours accurately before surgery, because preoperative therapeutic plans for more aggressive therapeutic management must be formulated to reduce the recurrence rate and possible tumour residues ${ }^{(14,25-27)}$. Pathological findings are the gold standard for diagnosis. Imaging guidance is crucial to improve the exact location of preoperative biopsy. Furthermore, we must remain alert to the possibility of malignancy in cases where preoperative biopsy did not indicate malignancy. This study provides us with an effective method for this. In this study, we assessed the ability of preoperative CT and MRI, including DCE-MRI, to predict SNIP-SCC as compared with postoperative pathology. Of the three independent risk factors identified, the nomogram model had the best predictive value for malignant transformation of SNIP.

Previous studies have found that bone erosion on CT is sugges- 


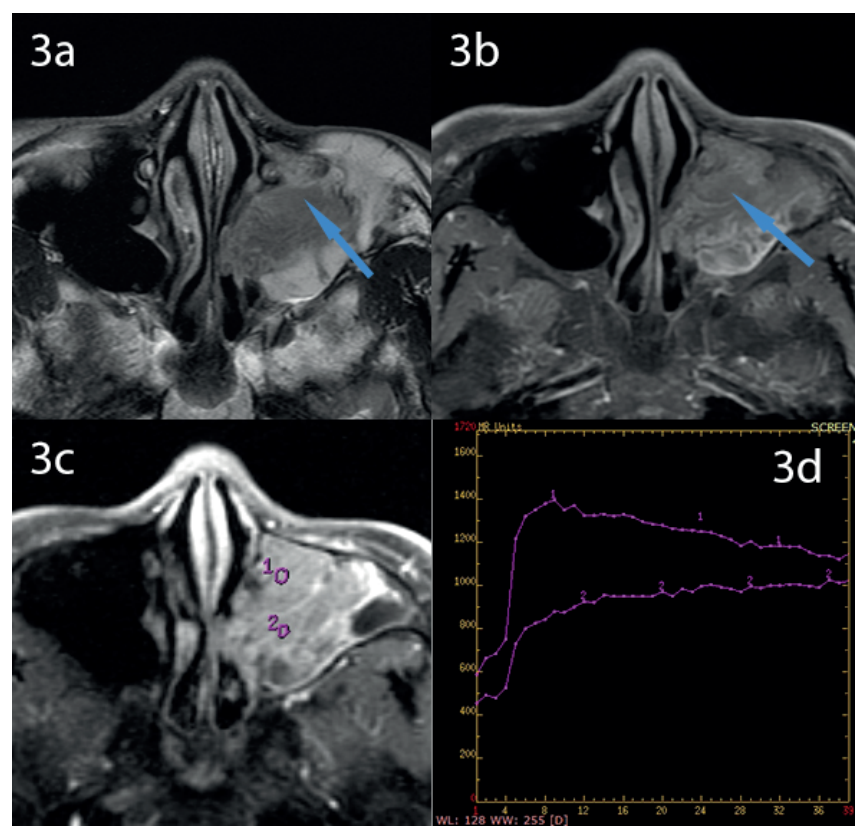

Figure 3. MRI of a 52-year-old man with left maxillary sinus lesions. The malignant transformation site of SNIP was indicated by blue arrows on $3 \mathrm{a}(\mathrm{T} 2 \mathrm{WI})$ and $3 \mathrm{~b}$ (contrast-enhanced $\mathrm{T} 1 \mathrm{WI}$ ) axial images. The 1 and 2 points on 3 c images were the perfusion sites of DCE-MRI, and the 1 and 2 points correspond to the 1 (washout-type) and 2 (persistent-type) TIC curves on $3 \mathrm{~d}$ images, respectively. Pathological examination showed that site 1 was SNIP-SCC and site 2 was SNIP. TIC = time-intensity curve; SNIP = sinonasal inverted papilloma; SNIP-SCC = SNIP-transformed squamous cell carcinomas; $M R I=$ magnetic resonance imaging; $D C E-M R I=$ dynamic contrast enhanced MRI.

tive of aggressive tumours, consistent with the transformation of SCC ${ }^{(28)}$. Bone erosion played an important role in predicting malignancy in this study. The AUC of bone erosion for predicting SNIP-SCC was 0.826 (95\% Cl: 0.761-0.890), with a sensitivity and specificity of $80.3 \%$ and $87.2 \%$, respectively. It also had a high score (10 points) according to the nomogram model. However, previous studies have shown that SNIP is also associated with bone erosion; thus, this feature alone is not enough to predict SNIP-SCC ${ }^{(29)}$.

The term CCP was proposed by Ojiri and colleagues ${ }^{(12)}$ to describe the features of SNIP, which manifests as alternating hypo- and hyperintense bands on T2W and enhanced T1W MRI. Subsequently, numerous reports of the characteristics of CCP for IP have been published ${ }^{(11-15,30-32)}$, and the disappearance of CCP indicates malignant transformation ${ }^{(15)}$. However, based on previous research results, only $50 \%$ of patients with SNIP-SCC demonstrated a partial loss of CCP, and the sample size was very small ${ }^{(15)}$. In our study, the AUC of CCP mutation for predicting malignant transformation of SNIP was 0.810 (95\% Cl: 0.7420.877 ) with a sensitivity and specificity of $73.4 \%$ and $85.4 \%$, respectively. A relatively high score of 7 points was obtained in the

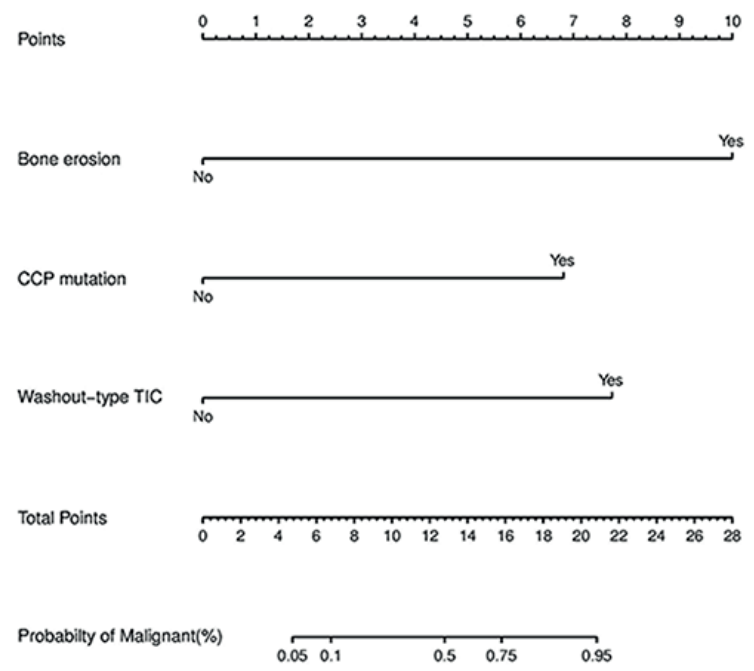

Figure 4. Nomogram for predicting malignant transformation of SNIP. To use the nomogram, the value of every indicator is located on each variable axis, and a line uppermost is drawn to determine the number of points derived from the value of each variable. The sum of these numbers is located on the total point axis, and a line is drawn downward to the axes to predict the possibility of malignant transformation of SNIP.

prediction of malignant transformation of SNIP, by means of the score of the nomogram model. However, CCP is limited in the context of benign papilloma, such as the small size of tumours or the location of tumours in too narrow areas, and typical CCP can also appear with malignant IP ${ }^{(19)}$; thus, distinguishing SNIP-SCC from SNIP by only the existence and disappearance or mutation of CCP is also not sufficient.

Therefore, we used evaluation of DCE-MRI sequences, with TIC type, as a relatively objective method to differentiate SNIP-SCC from SNIP. Previous studies have shown that malignant tumours exhibit washout-type TIC ${ }^{(19)}$. DCE-MRI can dynamically observe the degree and manner of lesion enhancement, and can to a certain extent reflect the perfusion, vascular permeability, and extracellular space volume of the corresponding lesions. It is helpful for identification of benign and malignant lesions ${ }^{(19)}$. In this study, the AUC of the washout-type TIC for predicting SNIP-SCC was 0.776 (95\% Cl: 0.703-0.848) and the sensitivity and specificity were $61.8 \%$ and $93.7 \%$, respectively. A high score of 8 was obtained for washout-type TIC for predicting malignancy of SNIP according to the nomogram model. Although the sensitivity for predicting SNIP-SCC is not very high, the specificity was very high; thus, malignant tumours may not necessarily appear to be washout-type TIC, but if the washout-type TIC is present, it certainly indicates malignant transformation, and has good clinical value for predicting SNIP-SCC.

In this study, a nomogram was constructed to evaluate the predictive power for SNIP-SCC in this study. The nomogram model, based on bone erosion on CT, CCP mutation on MRI, and was- 


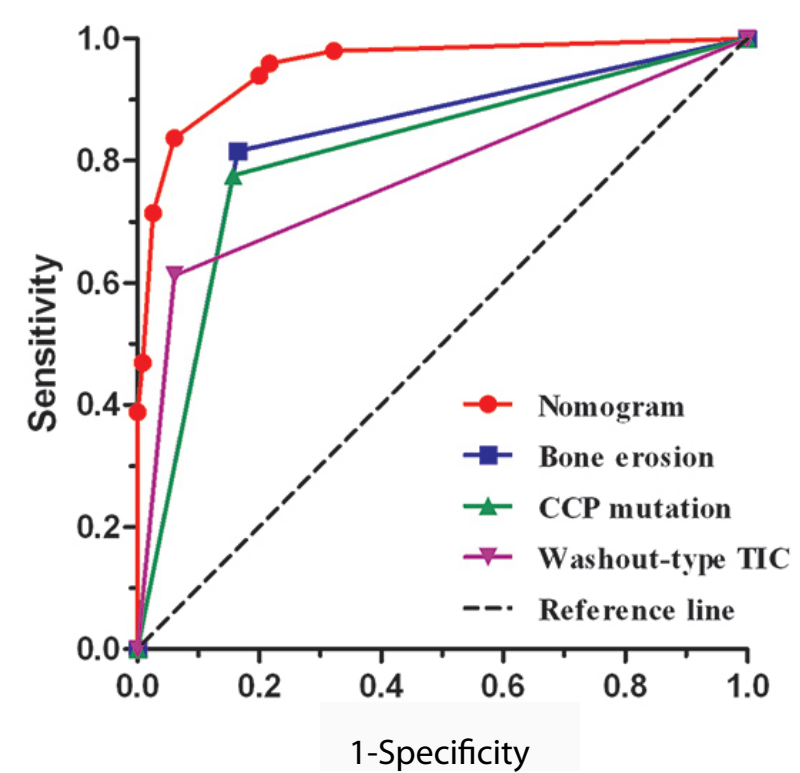

Figure 5. ROC curve analysis for predicting malignant transformation of SNIP with nomogram, bone erosion, CCP mutation and washout-type TIC. The predictions of malignant transformation of SNIP by nomogram are much higher than those of the three risk factors mentioned above by comparing the AUC area.

hout-type TIC on DCE-MRI, demonstrated superior predictive capability and clinical usefulness of the nomogram as compared to previous predictive methods. The AUC of the nomogram for predicting SNIP-SCC was 0.954 (95\%Cl: 0.920-0.987), with a sensitivity and specificity of $83.7 \%$ and $93.9 \%$, respectively. When the above three risk factors for SNIP-SCC coexist, a high score of 25 points was obtained according to the nomogram model, and the accuracy rate of predicting SNIP-SCC was more than $95 \%$. Our results indicated that older age, lack of involvement of the nasal cavity, and involvement of maxillary sinus are associated with malignant transformation of SNIP $(p<0.05)$. SNIP-SCC occurred more frequently in aged patients $(p=0.043)$ in this study. However, previous studies found no significant difference in the age of patients between those with IP and malignant tumours $(19,28,33)$. The inconsistent results may be due to malignancy mainly arising from incomplete operation, repeated surgical stimulation. and recurrence; thus, the age of patients with SNIP-SCC is older than that of non-malignant cases in this study. Differences in the invasive sites of tumours between SNIP and SNIP-SCC are probably related to the origin of the lesion. Based on previous research results, SNIP is common in the nasal cavity, involving the lateral wall of the nasal cavity (68\%-90\%) ${ }^{(9,34)}$ and carcinomas most often originate from the maxillary sinuses, followed by the ethmoid sinuses, nasal vestibule, and cavity ${ }^{\left({ }^{10}\right)}$. However, the above findings are uncertain and nonspecific, and to some extent it is inconsistent with previous research results. In this study, there were significant differences in recurrence,

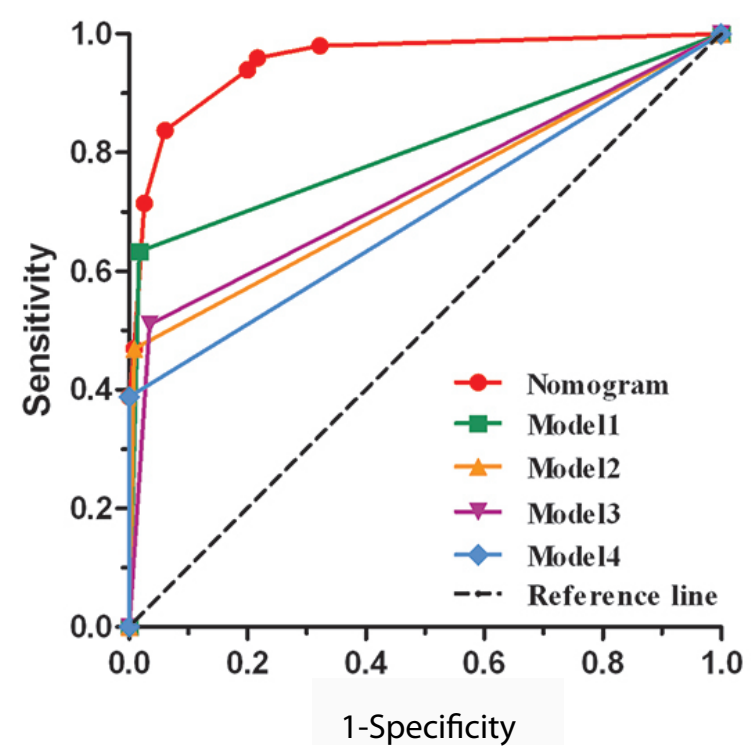

Figure 6. ROC curve analysis for predicting malignant transformation of SNIP with nomogram, and related combinations. Model $1=$ Bone erosion +CCP mutation, Model 2 = Bone erosion + Washout-type TIC, Model 3 = Washout-type TIC + CCP mutation, Model $4=$ Bone erosion + CCP mutation +Washout-type TIC. The predictions of malignant transformation of SNIP by nomogram are much higher than the above related combinations by comparing the AUC area.

extranasal invasion, and T2 signal strength between SNIP-SCC and SNIP $(p<0.01)$. Although these factors were not entered into the final regression analysis, they were also valuable for distinguishing SNIP-SCC from SNIP in clinical practice. Some malignancy is related to repeated surgical stimulation and incomplete resection in SNIP. Lee et al. stressed that, if SNIP is removed completely initially, there will be very few malignant changes ${ }^{(9)}$. Thus, there is a strong correlation between malignant change and recurrence. Extranasal invasion and $\mathrm{T} 2$ signal strength were closely related to bone erosion and variation of CCP, but the influence of the former two factors was weaker than that of the latter in the statistical analysis, which is why extranasal invasion and $\mathrm{T} 2$ signal strength, although important factors in predicting malignancy, were not included in the final regression model. In this study, we did not consider other findings of CT and MRI especially calcification and enhancement for the following reasons: first, both malignant and non-malignant inverted papilloma can cause bone hyperplasia and calcification, the occurrence of which was similar between the two groups $(40.7 \%(66 / 162)$ in SNIPs and 43.9\% (29/66) in SNIP-SCC group on CT). Second, both SNIP and SNIP-SCC have different degrees of enhancement on MRI. Especially in dynamic MRI enhancement studies, the inflow and outflow velocities of the contrast agents differ due to variability of blood vessel constriction, which leads to differences in the dynamic enhancement curves. This difference is an impor- 
tant index for the diagnosis and differentiation of canceration. There are some limitations in this study. First, recurrent tumours of this study could not fully reflect the initial state of the tumours, and the growth of new tumours along the bone defect caused by surgery is easily misattributed to being caused by the tumours themselves. Furthermore, surgery is generally performed in outside hospitals and surgical records are not necessarily complete, and thus, there may inevitably be errors that artificially expand the bone erosion caused by real tumours. Second, the three important indicators identified in the present study were all qualitative and subjective, which may lead to errors and imprecision, and this study was based on observations by two people, but no other consistency analysis was carried out to eliminate errors.

\section{Conclusion}

In conclusion, the combination of CT and MRI including DCEMRI is valuable for distinguishing SNIP-SCC from SNIP. The significant differences include 1) bone erosion on CT; 2) CCP mutation, TIC curves, and T2 signal strength on MRI; and 3) lack of involvement of the nasal cavity, involvement of maxillary sinus, extranasal invasion on MRI and/or CT. Ultimately, this is a qualitative and quantitative method that combines bone erosion, CCP mutation, and TIC curves in the nomogram model and corresponding scores to distinguish SNIP-SCC from SNIP. We recommend that dynamic MRI with TIC be obtained before surgery as part of routine SNIP imaging.

\section{Acknowledgements}

This work was supported by grants from the national key R\&D program of China (2016YFC0905200, 2018YFC0116801), the program for the Changjiang scholars and innovative research team (IRT13082), the national natural science foundation of China (81630023, 81870698), Beijing municipal administration of hospitals' mission plan (SML20150203), Beijing Municipal Administration of Hospitals Clinical Medicine Development of Special Funding Support (XMLX201816).

\section{Authorship contribution}

LZ: Design, acquisition of data, analysis, and interpretation of data, drafting of the manuscript, approval of final draft. GF: Design, acquisition of data, analysis, and interpretation of data, drafting of the manuscript, approval of final draft. WY: Analysis and interpretation of data, revision of the manuscript, approval of final draft. BY: Analysis and interpretation of data, revision of the manuscript, approval of final draft. CW: Design, acquisition of data, analysis, and interpretation of data, drafting of the manuscript, approval of final draft. LZ: Design, acquisition of data, analysis, and interpretation of data, drafting of the manuscript, approval of final draft.

\section{Conflict of interest}

None of the authors have any potential financial conflict of interest related to this manuscript.

\section{References}

1. Strojan P, Ferlito A, Lund VJ, et al. Sinonasa inverted papilloma associated with malignancy: the role of human papillomavirus infection and its implications for radiotherapy. Oral Oncol 2012; 48: 216-218.

2. Hong $\mathrm{SL}$, Kim BH, Lee $\mathrm{JH}$, Cho KS, Roh $\mathrm{HJ}$. Smoking and malignancy in sinonasal inverted papilloma. Laryngoscope 2013; 123: 1087-1091.

3. Busquets JM, Hwang PH. Endoscopic resection of sinonasal inverted papilloma: a meta-analysis. Otolaryngol Head Neck Surg. 2006; 134: 476-482.

4. Nygren A, Kiss K, von Buchwald C, Bilde A. Rate of recurrence and malignant transformation in 88 cases with inverted papilloma between 1998-2008. Acta Otolaryngol. 2016; 136: 333-336.

5. Krouse $\mathbf{J H}$. Endoscopic treatment of inverted papilloma: safety and efficacy. Am J Otolaryngol 2001; 22: 87-99.

6. Mirza S, Bradley PJ, Acharya A, Stacey M, Jones NS. Sinonasal inverted papillomas: recurrence, and synchronous and metachronous malignancy. J Laryngol Otol 2007; 121: 857-864.

7. Karligkiotis A, Lepera D, Volpi L, et al Survival outcomes after endoscopic resec- tion for sinonasal squamous cell carcinoma arising on inverted papilloma. Head Neck 2016; 38: 1604-1614.

8. Han MW, Lee BJ, Jang YJ, Chung YS. Clinical value of office-based endoscopic incisional biopsy in diagnosis of nasal cavity masses. Otolaryngol Head Neck Surg 2010; 143: 341-347.

9. Lee DK, Chung SK, Dhong HJ, Kim HY, Kim $\mathrm{HJ}$, Bok KH. Focal hyperostosis on CT of sinonasal inverted papilloma as a predictor of tumour origin. AJNR Am J Neuroradiol 2007; 28: 618-621.

10. Eggesbø HB. Imaging of sinonasal tumours Cancer Imaging 2012; 12: 136-152.

11. Yousem DM, Fellows DW, Kennedy DW Bolger WE, Kashima H, Zinreich SJ. Inverted papilloma: evaluation with MR imaging. Radiology 1992; 50: 472-475.

12. Savy L, Lloyd G, Lund VJ, Howard D. Optimum imaging for inverted papilloma. J Laryngol Otol 2000; 114: 891-893.

13. Maroldi R, Farina D, Palvarini L, Lombardi $D_{\text {, }}$ Tomenzoli D, Nicolai P. Magnetic resonance imaging findings of inverted papilloma: differential diagnosis with malignant sinonasal tumours. Am J Rhinol 2004; 18: 305-310.

14. Ojiri H, Ujita M, Tada S, Fukuda K. Potentially distinctive features of sinonasal invert- ed papilloma on MR imaging. AJR Am J Roentgenol 2000; 175: 465-468.

15. Jeon TY, Kim HJ, Chung SK, et al. Sinonasal inverted papilloma: value of convoluted cerebriform pattern on MR imaging. AJNR Am J Neuroradiol 2008; 29: 1556-1560.

16. Jeon TY, Kim HJ, Choi JY, et al. 18F-FDG PET/ CT findings of sinonasal inverted papilloma with or without coexistent malignancy: comparison with MR imaging findings in eight patients. Neuroradiology.2009; 51: 265-271.

17. Limura J, Otori N, Ojiri H, Moriyama $H$. Preoperative magnetic resonance imaging for localization of the origin of maxillary sinus inverted papillomas. Auris Nasus Larynx. 2009; 36: 416-421.

18. Jeon TY, Kim HJ, Chung S-K, et al. Sinonasal inverted papilloma: value of convoluted cerebriform pattern on MR imaging. AJNR Am J Neuroradiol 2008; 29: 1556-1560.

19. Wang $X Y$, Zhang $Z Y$, Chen $X L$, Li J, Xian J. Value of magnetic resonance imaging including dynamic contrast enhanced magnetic resonance imaging in differentiation between inverted papilloma and malignant tumours in the nasal cavity. Chin Med J. 2014; 127: 1696-1701.

20. Touijer K, Scardino PT. Nomograms for stag- 
ing, prognosis, and predicting treatment outcomes. Cancer. 2009; 115: 3107-3111.

21. Kattan MW, Scardino PT. Evidence for the usefulness of nomograms. Nat Clin Pract Urol. 2007; 4: 638-639.

22. Wan G, Gao F, Chen J, et al. Nomogram prediction of individual prognosis of patients with hepatocellular carcinoma. BMC Cancer. 2017; 17: 91.

23. Sternberg CN. Are nomograms better than currently available stage groupings for bladder cancer. J Clin Oncol. 2006; 24: 3819-3820.

24. Frank E, Harrell J. Rms: Regression Modeling Strategies. R Package version 3.4-0.

25. Lawson W, Kaufman MR, Biller HF. Treatment outcomes in the management of inverted papilloma: an analysis of 160 cases Laryngoscope 2003; 113:1548-1556.

26. Roobottom CA, Jewell FM, Kabala J. Primary and recurrent inverting papilloma: appearances with magnetic resonance imaging. Clin Radiol 1995; 50:472-475.

27. Pasquini E, Sciarretta $V$, Farneti G, Modugno GC, Ceroni AR. Inverted papilloma: report of 89 cases. Am J Otolaryngol 2004; 25:178185.

28. Miyazaki T, Haku Y, Yoshizawa A, et al. Clinical features of nasal and sinonasal inverted papilloma associated with malignancy. Auris Nasus Larynx 2018; 45: 1014
1019

29. Karkos PD, Fyrmpas G, Carrie SC, Swift AC. Endoscopic versus open surgical interventions for inverted nasal papilloma: a systematic review. Clin Otolaryngol 2006; 31 499-503.

30. Karkos PD, Khoo LC, Leong SC, Lewis-Jones $\mathrm{H}$, Swift AC. Computed tomography and/ or magnetic resonance imaging for preoperative planning for inverted nasal papilIoma: review of evidence. J Laryngol Otol 2009; 123: 705-709.

31. Fujima N, Nakamaru $Y$, Sakashita $T$, et al. Differentiation of squamous cell carcinoma and inverted papilloma using non-invasive MR perfusion imaging. Dentomaxillofac Radiol 2015; 44: 20150074.

32. Fang G, Lou H, Yu W, et al. Prediction of the originating site of sinonasal inverted papilloma by preoperative magnetic resonance imaging and computed tomography. Int Forum Allergy Rhinol 2016; 6: 1221-1228.

33. Yan $\mathrm{CH}$, Tong $\mathrm{CCL}$, Penta $\mathrm{M}$, et al. Imaging predictors for malignant transformation of inverted papilloma. Laryngoscope. 2019; 129: 777-782.

34. Minovi A, Kollert M, Draf W, Bockmühl U. Inverted papilloma-feasibility of endonasal surgery and long-term results of 87 cases. Rhinology 2006, 44: 205-210.
Luo Zhang, M.D., Ph.D.

Beijing TongRen Hospital

Capital Medical University

No. 1, DongJiaoMinXiang

DongCheng District

Beijing

China

Tel: +86-13910830399

E-mail:dr.luozhang@139.com

Chengshuo Wang, M.D., Ph.D.

Beijing TongRen Hospital

Capital Medical University

No. 1, DongJiaoMinXiang

DongCheng District

Beijing

China

E-mail: wangcs830@126.com 